\title{
Polarization properties of standing shear Alfvén waves in non-axisymmetric background magnetic fields
}

\author{
K. Kabin, R. Rankin, I. R. Mann, A. W. Degeling, and R. Marchand \\ Department of Physics, the University of Alberta, Edmonton, Alberta, T6G 2G7 Canada
}

Received: 23 November 2006 - Revised: 26 February 2007 - Accepted: 27 February 2007 - Published: 29 March 2007

\begin{abstract}
In this paper we present results concerning periods and polarizations of cold plasma ultra-low frequency (ULF) guided Alfvén waves in a non-axisymmetric geomagnetic field. The background geomagnetic field is approximated by a compressed dipole for which we propose a simple description in terms of Euler potentials. This study is motivated by the problem of outer-radiation belt electron acceleration by ULF waves, for which the polarization of the wave is of paramount importance. We consider an approximation appropriate to decoupled Alfvénic waves and find that the polarization of the waves can change significantly with local time. Therefore, the ULF wave's contribution to the MeV electron energization process can be localized in space.
\end{abstract}

Keywords. Magnetospheric physics (Energetic particles, trapped; MHD waves and instabilities) - Space plasma physics (Kinetic and MHD theory; Wave-particle interactions)

\section{Introduction}

It has been long accepted (Rostoker et al., 1998; Mathie and Mann, 2000; Elkington et al., 2003; O'Brien et al., 2003; Elkington, 2006) that one of the mechanisms potentially responsible for the acceleration of electrons in the outer radiation belts is their interaction with ultra-low frequency (ULF) waves in the Pc 5 frequency range. The effectiveness of this acceleration mechanism depends crucially on the polarization of the waves, since the necessary condition for energy transfer from the wave to the particle is that the wave has an electric field component parallel to the drift velocity of the particle (e.g. Northrop, 1963, p. 10). Thus, in dipolar background magnetic field ULF waves with an azimuthal electric field (referred to as poloidal modes, since the usual classifi-

Correspondence to: K. Kabin

(kabin@phys.ualberta.ca) cation is based on the wave's magnetic field polarity, rather than on that of the electric field) contribute to particle acceleration while the waves with a radial electric field (toroidal modes) do not.

Elkington et al. (2003) considered energization of $\mathrm{MeV}$ energy electrons by ULF waves in a non-axisymmetric compressed dipole model for the magnetosphere. They restricted their study to equatorially mirroring particles only and assumed, somewhat simplistically, that the electric field of the ULF waves was either exactly radial or exactly azimuthal and was characterized by a single frequency. In the absence of ULF waves, conservation of the magnetic moment of the particles requires that, to first order, electrons in the equatorial plane drift along contours of constant magnetic field. Thus, the acceleration by ULF waves in the model of Elkington et al. (2003) appears simply as a geometrical effect, since the lines of constant magnetic field are no longer circles. In fact, however, the polarization of the ULF waves has to be computed self-consistently and may differ from either purely radial or azimuthal. Elkington et al. (2003) also implicitly assumed that the frequencies of poloidal and toroidal waves are the same, which is usually not the case, at least not for the fundamental mode (e.g. Cummings et al., 1969). The assumptions of Elkington et al. (2003) are perfectly reasonable and natural if one concentrates on the two-dimensional dynamics of the equatorial electrons. However, recent progress in ULF wave modeling allows us to assess those assumptions more carefully, and to refine some of them.

In this paper we propose a novel three-dimensional generalization of the two-dimensional compressed dipole field used by Elkington et al. (2003). Our three-dimensional background magnetic field is described in terms of Euler potentials (e.g. Stern, 1970), which makes it particularly convenient for studies which require knowledge of the flux surfaces (e.g. D'haeseleer et al., 1991; Northrop, 1963). The compressed dipole model includes interplanetary magnetic field (IMF), open, and closed field lines and somewhat

Published by Copernicus GmbH on behalf of the European Geosciences Union. 
realistically mimics the compression of the day-side magnetosphere and stretching of the magnetotail on the nightside. Next, we apply to this field a recently developed standing shear Alfvén wave model (Rankin et al., 2006) which allows us to compute self-consistently both the frequency and polarization of the standing shear Alfvén wave in the cold plasma approximation. This allows us to test, for the first time, some of the assumptions used by Elkington et al. (2003). Not surprisingly, we find in contrast to the assumptions of Elkington et al. (2003) that the contours of constant magnetic field do not coincide with the contours of constant frequency of the free oscillations of the field lines. More importantly, we find that the polarizations of the decoupled Alfvén wave modes can deviate significantly from the pure radial or pure azimuthal modes assumed by Elkington et al. (2003). As the degree of asymmetry of the background magnetic field increases, these waves can no longer be described as either poloidal or toroidal and exhibit increasingly mixed characteristics. Polarization properties of a single mode of the ULF Alfvén waves also change with magnetic local time (MLT), so that waves with equatorial electric field essentially perpendicular to the particle trajectory in some sectors (and, therefore, contributing little to the energization of the outer radiation belt electrons) but may have a significant electric field component along the particle trajectory in other sectors (where they may effectively contribute to particle energization).

Note that in models with a more realistic time-dependence for toroidal Alfvén wave excitation by fast mode waves, the local polarization at the resonance evolves with time (e.g. Mann et al., 1995). Similarly, for the excitation of dominantly poloidal Alfvén waves there is an important timedependent evolution of wave polarization (Mann and Wright, 1995). The results presented here are applicable to the case where any coupling to a driving fast mode is neglected. Our analysis generates two Alfvénic wave modes with different polarization characteristics representing the compressed dipole eigensolutions. These two modes are generalizations of the solutions to two field aligned eigenvalue problems in the dipole case which correspond to the zero and infinite azimuthal wave number limits, respectively (e.g. Radoski, 1967).

\section{Compressed dipole}

In this section, we discuss one possible three-dimensional generalization of the equatorial compressed dipole magnetic field used by Elkington et al. (2003). Such a generalization is necessary in order to consider standing shear Alfvén waves along geomagnetic field lines. We suggest an Euler potentials description of the field $\mathbf{B}=\nabla \alpha \times \nabla \beta$ with

$\alpha=\frac{B_{0}}{r} \sin ^{2} \theta-\frac{1}{m} r^{2} b_{1}\left(1+b_{2} \cos \phi\right) \sin ^{m} \theta, \beta=\phi$
Here $r, \theta, \phi$ are the usual spherical coordinates with $r$ measured in Earth radii and $B_{0}$ is the equatorial strength of the dipole field at $r=1$. The parameters $b_{1}$ and $b_{2}$ describe the distortion of the dipole field: $b_{1}$ can be interpreted as a quantity related to the IMF strength and $b_{2}$ is a non-dimensional parameter responsible for the azimuthal asymmetry of the resulting field (influenced, to a large degree, by the solar wind dynamic pressure). The parameter $m$ can, in principle, be arbitrary (subject to $m \geq 2$ to avoid a singularity in the magnetic field along the dipole axis), however we set it to $m=2$ in the following study as this choice gives a realistically looking field and has an additional advantage of simplifying the algebra.

The above expressions for the Euler potentials represent an obvious generalization of the well-known expressions for a dipole field, with which they coincide if $b_{1}=0$. As $r$ becomes small, the magnetic field asymptotically approaches that of a dipole field. Magnetic field topology in this compressed dipole is the same as in vacuum superposition models as described, for example, by Cowley (1973).

The magnetic field corresponding to Euler potentials (Eq. 1) is

$$
\begin{aligned}
\mathbf{B}= & \mathbf{e}_{r}\left(\frac{2 B_{0}}{r^{3}}-b_{1}\left(1+b_{2} \cos \phi\right)\right) \cos \theta \\
& +\mathbf{e}_{\theta}\left(\frac{B_{0}}{r^{3}}+b_{1}\left(1+b_{2} \cos \phi\right)\right) \sin \theta
\end{aligned}
$$

At the equator $(\theta=\pi / 2)$ this field reduces to

$B(r, \phi)=\frac{B_{0}}{r^{3}}+b_{1}\left(1+b_{2} \cos \phi\right)$

which is the same as the compressed dipole of Elkington et al. (1999, 2003). Of course, since Elkington et al. (2003) only considered the equatorial plane, the question of whether the field lines were closed or not did not arise in their study. In our three-dimensional version of the compressed dipole field, there is a boundary between closed and open field lines (see Fig. 1), which is a feature common to all vacuum superposition models with non-zero IMF (Cowley, 1973). In the equatorial plane, however, our open/closed field line boundary corresponds to the boundary between trapped and unbounded particle motions which is suggested by a singularity in $r$ appearing in Eq. (2) of Elkington et al. (2003); note an obvious typo in their original manuscript. At noon, this boundary is located at $r=\left(B_{0} / b_{1} b_{2}\right)^{1 / 3}$.

Field lines for this magnetic field lie in the $\phi=$ const planes, so the torsion of the field lines is zero. However, the field is clearly not axisymmetric. The equation of a field line for a particular $\phi$ and $\alpha_{0}$ is

$$
\sin ^{2} \theta(r)=\frac{\alpha_{0} r}{B_{0}-\frac{1}{2} r^{3} b_{1}(1+\cos \phi)} .
$$

If instead, a more familiar expression $r(\theta)$ is desired, it needs to be computed numerically. 


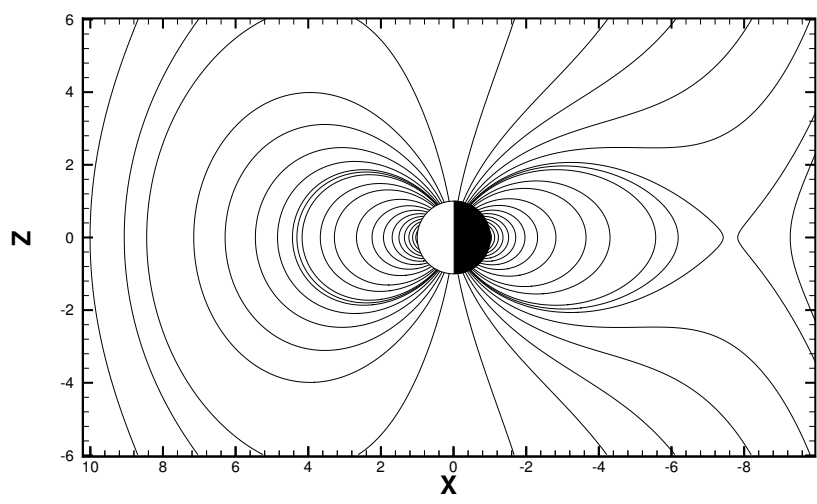

Fig. 1. Field lines of a compressed dipole in the noon-midnight meridional plane for $B_{0}=31000 \mathrm{nT}, b_{1}=10 \mathrm{nT}, b_{2}=8$.

Field lines (contours of constant $\alpha$ ) for the compressed dipole in the noon-midnight meridional plane with parameters $B_{0}=31000 \mathrm{nT}, b_{1}=10 \mathrm{nT}, b_{2}=8$ are shown in Fig. 1 . A three-dimensional view of field lines with the same parameters is shown in Fig. 2. The X-axis in this plot is pointing towards the Sun and the dipole axis in the Z-direction. The color code in this figure shows the magnetic field strength in the equatorial plane. The field lines shown in this figure are initialized along a contour of constant magnetic field strength in the equatorial plane (which is different from an $\alpha=$ const contour).

\section{Eigenmode equations}

For the compressed dipole field (Eq. 2), we define a curvilinear field-aligned non-orthogonal coordinate system $(\alpha, \beta, \mu)$. Here $\alpha$ and $\beta$ are the Euler potentials given by Eqs. (1), which are constant along any particular field line, and $\mu=\cos \theta / r^{2}$ is the third coordinate, which changes along the field line. For pure dipole field $\left(b_{1}=0\right)$ these coordinates become the usual dipole coordinates frequently used in studies of field line resonances (e.g. Cummings et al., 1969; Walker, 1980). We note that unless $b_{1}=0$ there is a non-zero component of $\nabla \times \mathbf{B}$ in the direction of the magnetic field and, therefore, an orthogonal field aligned coordinate system does not exist for such fields (Salat and Tataronis, 2000).

We follow the approach of Rankin et al. (2006) and write equations for the standing shear Alfvén wave as

$$
\begin{aligned}
& \frac{1}{\sqrt{g}} \frac{\partial \delta B_{2}}{\partial \mu}=\frac{1}{v_{A}^{2}}\left(g^{11} \omega \delta E_{1}+g^{12} \omega \delta E_{2}\right) \\
& \frac{1}{\sqrt{g}} \frac{\partial \delta B_{1}}{\partial \mu}=-\frac{1}{v_{A}^{2}}\left(g^{21} \omega \delta E_{1}+g^{22} \omega \delta E_{2}\right) \\
& \frac{1}{\sqrt{g}} \frac{\partial \delta E_{1}}{\partial \mu}=-\left(g^{12} \omega \delta B_{1}+g^{22} \omega \delta B_{2}\right)
\end{aligned}
$$

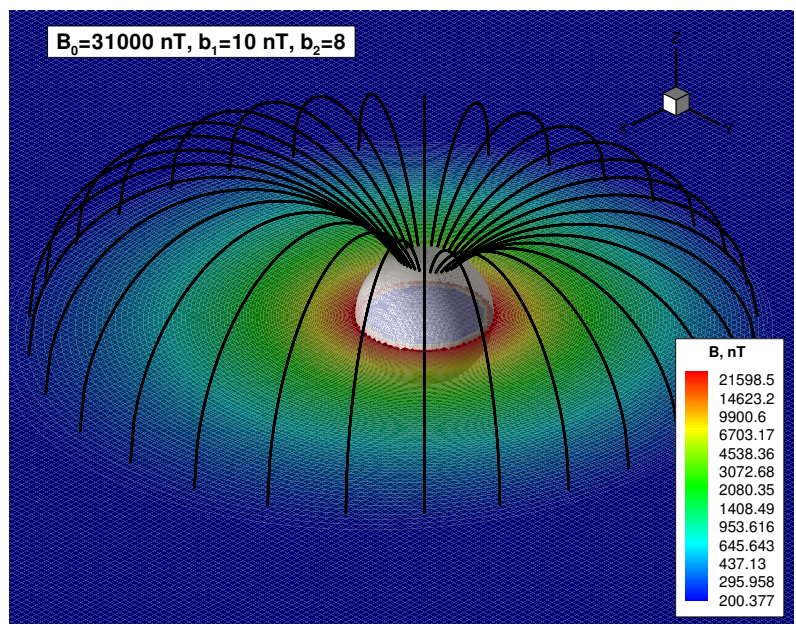

Fig. 2. Three-dimensional view of a compressed dipole field lines with $B_{0}=31000 \mathrm{nT}, b_{1}=10 \mathrm{nT}, b_{2}=8$. The color code shows the equatorial magnetic field intensity. The sphere represents the Earth $(r=1)$.

$$
\frac{1}{\sqrt{g}} \frac{\partial \delta E_{2}}{\partial \mu}=\left(g^{11} \omega \delta B_{1}+g^{12} \omega \delta B_{2}\right)
$$

In the uncoupled axisymmetric limit, these equations would describe two decoupled solutions for the toroidal (zero azimuthal wave number) and poloidal (infinite azimuthal wave number) guided Alfvén modes (e.g. Radoski, 1967). Here $g^{11}, g^{12}$, and $g^{22}$ are components of the metric tensor and $g$ is the determinant of the inverse tensor (Borisenko and Tarapov, 1968) for the field-aligned coordinate system. These parameters have to be evaluated from gradients of the coordinate functions at every point along the field line. For this system of equations, we need to solve a two-parameter eigenvalue problem for the period of the standing Alfvén wave and its polarization at one of the ionospheres. The polarization of the wave at any other point along the field line is then computed from the corresponding eigenfunctions, see Rankin et al. (2006) for details. This approach to computing Alfvénic frequencies of the field lines and polarizations of the standing shear Alfvén waves is more self-consistent than the commonly used approach of Singer et al. (1981), in which the polarizations of the waves have to be imposed explicitly. The Singer et al. (1981) model, furthermore, implicitly relies on a field-aligned orthogonal coordinate system, which may not exist in general. A more detailed comparison of various models for standing shear Alfvén waves can be found in (Kabin et al., 2007). Note that in general the solutions of this standing Alfvén wave model based on Eqs. (36) cannot be clearly classified as either toroidal or poloidal, but instead have hybrid characteristics. In fact, in nonaxisymmetric background fields, the definitions of poloidal and toroidal directions become ambiguous, and we prefer to refer to the wave polarizations in the equatorial plane as either 

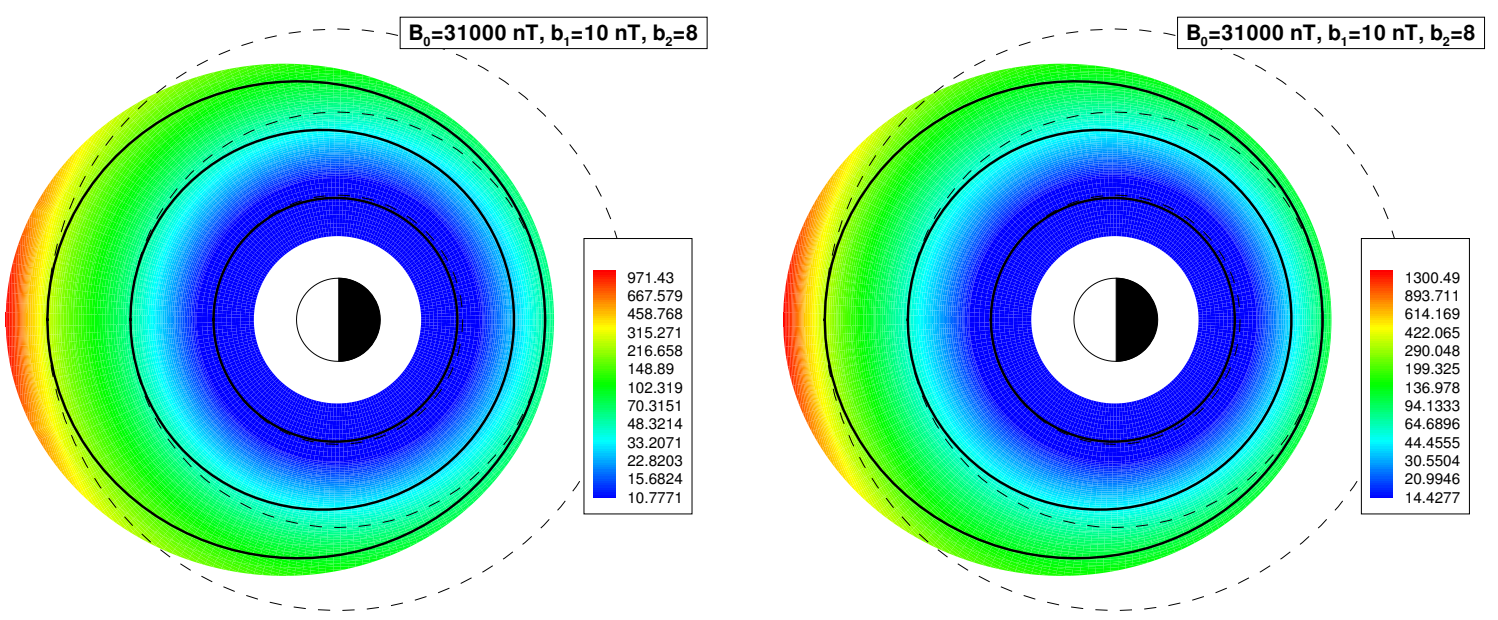

Fig. 3. Periods of the two different Alfvén modes for $B_{0}=31000 \mathrm{nT}, b_{1}=10 \mathrm{nT} b_{2}=8$. Left panel: mode with radial electric field polarization at midnight; right panel - with azimuthal electric field at midnight. Thick solid lines show the contours of constant $B$ initiated at $r=2,4$, and 6 at noon. Dashed lines are circles with $r=2,4$, and 6 , respectively.

radial or azimuthal, or alternatively as parallel and perpendicular to the contours of constant $B$, as the discussion requires. We note that in the particular case of compressed dipole background field, we have explicit expressions for the fieldaligned coordinate system as functions of the usual spherical coordinates. This greatly simplifies our calculations and makes many numerical steps described in the previous papers (Rankin et al., 2005, 2006; Kabin et al., 2007) unnecessary. The availability of analytical expressions for the gradients of the coordinates also allows us to minimize numerical errors.

In order to proceed with the calculations, we need to specify the background plasma density. In the context of the present study, the details of the density distribution are not important. For simplicity, we choose the density profile to be a power law (e.g. Waters et al., 1996) $\rho=\rho_{e q}(r / 5)^{-4}$, where $r$ is measured in Earth radii, and $\rho_{e q}=7 \mathrm{amu} / \mathrm{cm}^{3}$. Detailed studies of the effect of the density distribution on the ULF wave frequencies have been conducted in the past (e.g. Orr and Matthew, 1971).

Finally, we note that the noon-midnight plane is the plane of symmetry for the compressed dipole along which the azimuthal derivatives of the background field disappear. Therefore, along these two meridians, the $g^{12}$ coefficient in Eqs. (3-6) is zero, and they can be simplified. In fact, along the noon-midnight meridian the assumptions of Rankin et al. (2000) apply, and the eigenmode equations can be written as (Rankin et al., 2006):

$$
\begin{aligned}
& \frac{d^{2} \delta E_{1}}{d s^{2}}+\frac{d \delta E_{1}}{d s} \cdot \frac{d}{d s} \ln \left(B(s) R^{2}(s)\right)+\frac{\omega^{2}}{v_{A}^{2}} \delta E_{1}=0 \\
& \frac{d^{2} \delta E_{2}}{d s^{2}}-\frac{d \delta E_{2}}{d s} \cdot \frac{d}{d s} \ln \left(B(s) R^{2}(s)\right)+\frac{\omega^{2}}{v_{A}^{2}} \delta E_{2}=0
\end{aligned}
$$

Here, $R$ is the distance from the dipole axis and $s$ is the distance along the field line, both in Earth radii. The only two polarizations which can occur along the noon-midnight line are pure radial and pure azimuthal, and the two Alfvén wave modes are decoupled along these two meridians. This provides a useful check on our solutions for ULF waves in compressed dipole obtained with the general code.

\section{ULF wave properties in the compressed dipole geo- magnetic field}

In this section, we apply the methodology of Sect. 3 to the compressed dipole geometry of Sect. 2. Note that our ULF wave model does not include any excitation mechanism; it only describes the periods and polarizations of the Alfvénic oscillations of individual field lines assuming that there is no coupling between them. Our model also does not provide information about the amplitudes of the standing shear Alfvén waves nor how the wave fields change with radius and azimuthal angle. The eigensolutions considered in this work are generalizations of the well-known guided toroidal and poloidal modes which exist in dipole field and are generally interpreted as representing time-asymptotic states of the Alfvénic oscillations in the magnetosphere.

In considering the global field-aligned structure of modes of oscillations of magnetic field lines, we need to compute contours of constant period for both mode polarizations. These contours define the shells of field lines which would be excited by coherent fast mode global oscillations at a specific fixed frequency. For a particular choice of parameters, $B_{0}=31000 \mathrm{nT}, b_{1}=10 \mathrm{nT}, b_{2}=8$, Fig. 3 shows the distribution of periods in the equatorial plane. One can see that the contours of constant period do not follow contours of 


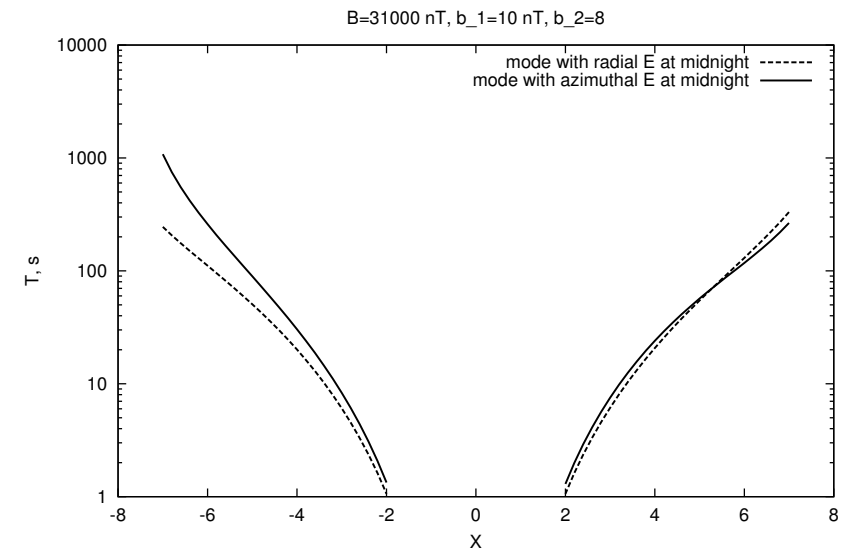

Fig. 4. Periods of the two Alfvén wave modes along noon-midnight line. Positive $X$ is towards the Sun.

constant magnetic field, since other parameters (such as the field line length and plasma density) affect the frequencies of the field lines. This modifies one of the assumptions used by Elkington et al. (2003). Furthermore, the constant period contours are different for the two mode polarizations (see also Fig. 5, discussed later, for a clear demonstration of this difference) so the magnetic shells supporting Alfvénic wave modes of given frequency but with different polarizations would not be the same. Since the contours of constant $B$ are the trajectories of equatorial particles conserving their magnetic moment, the fact that they are not contours of constant Alfvénic wave period implies that, in case of free Alfvénic oscillations of the magnetic field lines, the drifting particle will interact with waves characterized by a finite band of frequencies along its trajectory, rather than with a wave with a single frequency. In the case when the magnetic field oscillations are strongly driven by a fast mode source with a single frequency, the drifting particle will, of course, experience only the frequency of the driver. However, in this case, the amplitude of the forced oscillations will peak where the driving frequency coincides with the natural standing Alfvén wave frequency of the field line. Therefore, the electric field experienced by the particle as it drifts around the Earth will be strongly modulated, which obviously has ramifications for radial transport. The areas of enhanced radial transport associated with stronger electric fields will be localized in space, and in general, particle energization will be more similar to acceleration by a broad-band ULF wave package than by a single frequency ULF mode. Characteristics of such transport may be expected to appear diffusive after even a small amount of phase-space averaging (e.g. Schulz and Lanzerotti, 1974).

Figure 4 shows the Alfvén wave periods for the two modes with different polarizations along the noon-midnight line for the same parameters as before: $B_{0}=31000 \mathrm{nT}, b_{1}=10 \mathrm{nT}$, $b_{2}=8$ (with positive $X$ being towards the Sun). On the dayside where the dipole is compressed there is a point, around

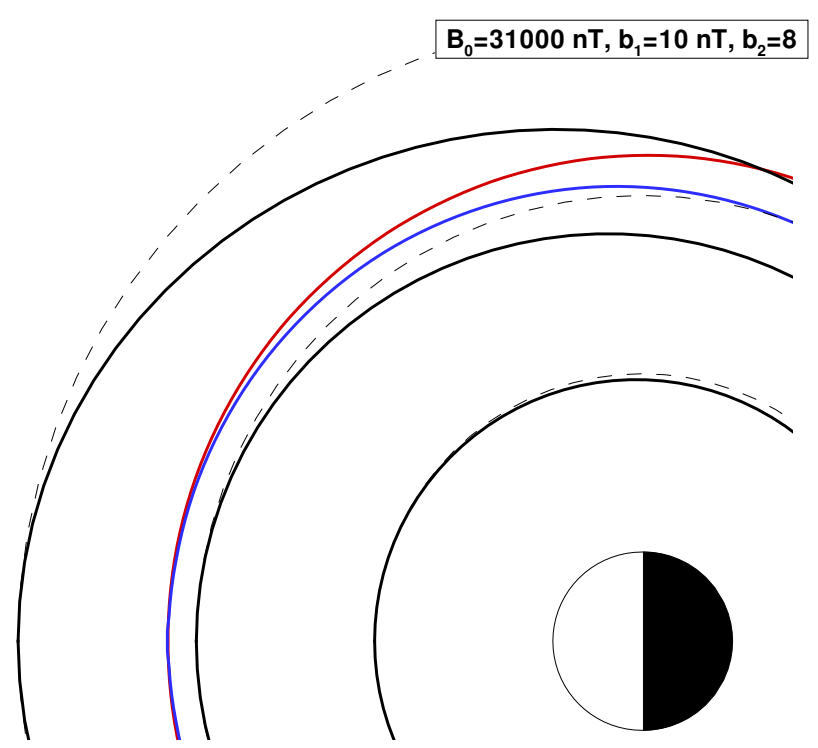

Fig. 5. Two contours of constant period (red - mode with azimuthal equatorial electric field at midnight, blue - mode with radial electric field at midnight) which intersect at a point on the noon meridian.

$x \approx 5$ where the periods of the two Alfvén waves modes with different polarizations are the same. Closer to the Earth than this point, the mode with the azimuthal equatorial electric field has a higher frequency than the one with the radial electric field (which is consistent with the usual results for a background dipole field). In contrast, on the night-side where the field lines are extended into the tail, the difference between the periods of the two modes increases when compared to the pure dipole case. Right at the point on the day-side where the two frequencies coincide, the system of Eqs. (3-6) becomes degenerate in the sense that the polarization of the standing Alfvén wave can be arbitrary. It also leads to numerical difficulties in finding such a solution, but fortunately this degeneracy occurs on the symmetry axis, where the simpler model of Rankin et al. (2006) based on Eqs. (7) and (8) applies. Furthermore, it turns out that the only point in the whole domain where the two frequencies intersect lies on the noon meridian. This is further illustrated by Fig. 5 which shows the two contours of the same period for the two Alfvénic modes with different polarizations which touch at noon and diverge from each other for MLT not equal to 12 . Therefore, a contour of constant period of one mode never intersects a contour of the same period of the other mode, except at a single point on the noon meridian where the modes become degenerate.

From the above discussion it follows that, if we consider, for example, a mode with azimuthal equatorial electric field at some point on the nightside, and trace it along a contour of constant period to the point on the day-side further from the Earth than the point where the two frequencies intersect, this mode will be characterized by a radial electric field at noon. 


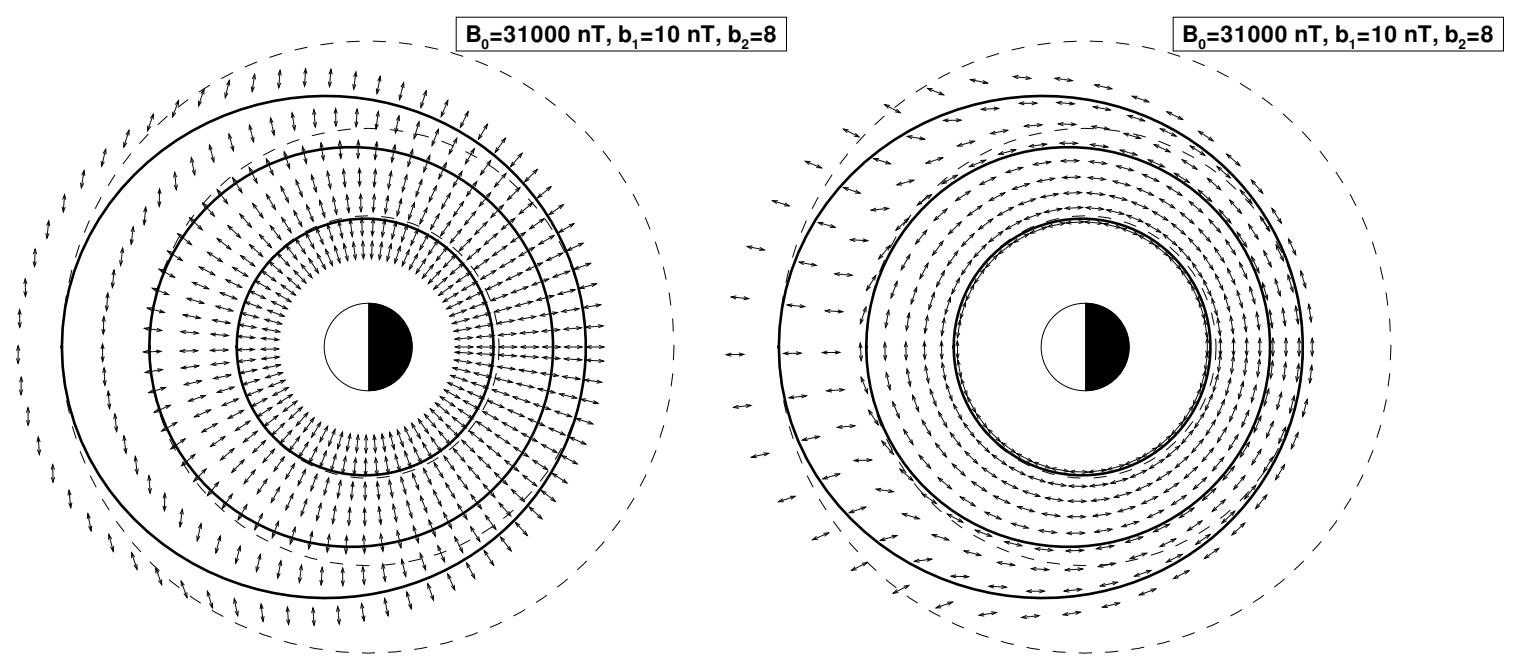

Fig. 6. Electric field polarizations in the equatorial plane for the two Alfvénic eigenmodes. Left panel: mode with radial electric field polarization at midnight; right panel - with azimuthal electric field at midnight. Thick solid lines show the contours of constant $B$ initiated at $r=3,5$, and 7 at noon. Dashed lines are circles of constant radius with $r=3,5$, and 7 . Note the sudden change of the polarization along the noon meridian (see text for details).

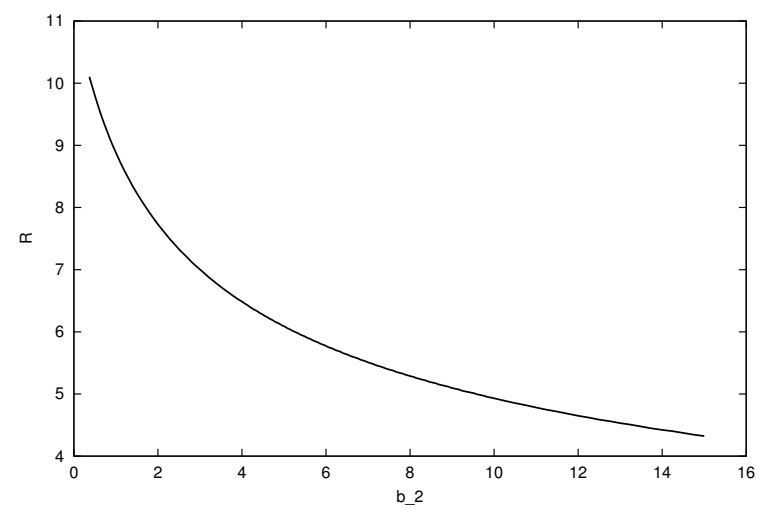

Fig. 7. Distance, as a function of $b_{2}$, to the point where periods of the two shear Alfvén modes coincide.

This is indeed supported by the calculations of the polarizations of the waves, shown in Fig. 6. This figure shows the equatorial polarizations of the standing Alfvén waves electric field for the two fundamental modes for the same parameters as used in the previous figures. Along the noon meridian there is a point where the polarization of the mode changes suddenly from azimuthal to radial (or vice-versa). As explained above, this sudden polarization change occurs exactly at the point where the periods of the two modes coincide. However, along any contour of constant period or a contour of constant background magnetic field the polarization varies smoothly. Since the noon-midnight plane is a plane of symmetry for the background magnetic field, only purely radial and purely azimuthal polarizations are possible along the noon-midnight meridian. Thus, should the polar- ization of the wave change along this line, this change can only be discontinuous since no intermediate angles of polarization are available (as long as the two fundamental frequencies are not exactly the same).

For different values of $b_{2}$, the behavior of the periods and polarizations of the two modes are qualitatively similar to the case $b_{2}=8$ presented in Figs. 1-6. Not surprisingly, the distance from the Earth to the point of intersection of the frequencies along the noon meridian increases as $b_{2}$ becomes smaller. Variation of this distance as a function of $b_{2}$ (holding $B_{0}=31000 \mathrm{nT}, b_{1}=10 \mathrm{nT}$ fixed) is shown in Fig. 7 .

The analysis above shows that the polarizations of ULF waves in a non-axisymmetric background magnetic field can change considerably with MLT. Since the polarization of the ULF waves is a primary factor determining their effectiveness in producing drift-resonance electron acceleration, these results have an important bearing on the drift resonance mechanism. Although this is outside the scope of the present paper, our results indicate that the region of effective interaction between radiation belt electron and ULF waves can be limited in space; they also open possibilities for electrons to be accelerated by the mostly "toroidal" mode, which is not usually considered to be effective. However, the detailed calculations of wave-particle interactions remain to be carried out. The next step required for such calculations is constructing a two-dimensional model (e.g. Degeling et al., 2007) which includes a fast magneto-acoustic wave as an excitation mechanism for the shear Alfvén waves examined in this work.

By comparing our results to the two solutions which exist for decoupled guided Alfvén wave modes in a dipole field, 
namely the toroidal (corresponding to zero azimuthal wave number) and poloidal (large azimuthal wave number) guided Alfvén wave modes (e.g. Radoski, 1967), we speculate that one of the Alfvén wave mode branches may be more easy to excite and may stay more closely coupled to global scale fast mode disturbances than the other. This suggests that the polarization of guided Alfvén waves which are excited by the solar wind might be a much stronger function of MLT than previously thought. If this is true, this has very significant implications for our understanding of the structure of ULF waves in a compressed magnetosphere. More importantly, if the polarization structure shown in Fig. 6 is maintained in the coupled wave solutions this will have a significant impact on the process of drift-resonance acceleration of $\mathrm{MeV}$ radiation belt electrons, most importantly introducing strong MLT asymmetry in the regions where the electrons and the ULF waves are most strongly coupled.

\section{Conclusions}

In this paper, we have proposed a three-dimensional generalization of the compressed dipole magnetic field model used by Elkington et al. (2003) and have analyzed the properties of standing shear Alfvén waves using a recently developed model of Rankin et al. (2006). We find that contours of constant magnetic field in the equatorial plane do not coincide with contours of constant periods for either of the two possible modes which differ by their polarizations. Therefore, an electron drifting approximately along a contour of constant magnetic field will interact, in the case of free Alfvénic oscillations of the field lines, with a packet of ULF waves with somewhat different frequencies, rather than with a single ULF mode. In the case of forced oscillations with a fixed driver frequency the electric field experienced by the electron is expected to be modulated by the condition of resonance between the driver and the natural frequencies of the field lines.

We find that for the considered background magnetic field, there is a point at local noon where the frequencies of the two modes with different polarizations coincide. This confluence of the frequencies, however, occurs only on the noon meridian, and does not happen for any other MLT.

Unexpectedly, we find that the equatorial polarizations of the two ULF wave modes in our model do not remain either radial or azimuthal but change continuously with MLT. In fact, the polarization of a single Alfvénic mode can change significantly as a function of MLT, and a mode for which the electric field is mostly radial for most MLTs may have a distinctly azimuthal electric field in an MLT sector centered around noon in our magnetic field model. Therefore, different Alfvénic modes may contribute to the acceleration of electrons in different MLT sectors. The variation of polarization with azimuth also means that the polarization properties of ULF waves inferred from either ground-based or satellite measurements at a certain MLT cannot be assumed to be the same as at other MLTs. Our analysis of standing Alfvénic waves in a compressed dipole geometry shows that several of the assumptions regarding the ULF wave properties used in the work of Elkington et al. (2003) are, in fact, quantitatively not accurate. Further study is, however, required to see if more realistic ULF wave properties will have any profound effect on the drift-resonant acceleration of the radiation belt electrons.

Acknowledgements. This work is supported by the Canadian Space Agency and by Natural Sciences and Engineering Research Council of Canada. We also acknowledge the use of WestGRID computational resources.

Topical Editor I. A. Daglis thanks S. R. Elkington and another referee for their help in evaluating this paper.

\section{References}

Borisenko, A. I. and Tarapov, I. E.: Vector and tensor analysis with applications, Dover Publication, New York, 1968.

Cowley, S. W. H.: A qualitative study of the reconnection between the Earth's magnetic field and interplanetary field of arbitrary orientation, Radio Sci., 8, 903-913, 1973.

Cummings, W. D., O'Sullivan, R. J., and Coleman, P. J.: Standing Alfvén waves in the magnetosphere, J. Geophys. Res., 74, 778793, 1969.

Degeling, A. W., Rankin, R., Kabin, K., Marchand, R., and Mann, I.: The effect of ULF compressional modes and field line resonances on relativistic electron dynamics, Planet. Space Sci., in press, doi:10.1016/j.pss.2006.04.039, 2007.

D'haeseleer, W. D., Hitchon, W. N. G., Callen, J. D., and Shohet, J. L.: Flux coordinates and magnetic field structure, SpringerVerlag, Berlin, Germany, 1991.

Elkington, S. R.: A review of ULF interactions with radiation belt electrons, in: Magnetospheric ULF Waves: Synthesis and New Directions, edited by: Takahashi, K., Chi, P. J., Denton, R. E., and Lysak, R. L., pp. 177-193, AGU monograph 169, 2006.

Elkington, S. R., Hudson, M. K., and Chan, A. A.: Acceleration of relativistic electrons via drift-resonant interaction with toroidalmode Pc-5 ULF oscillations, Geophys. Res. Lett., 26, $3273-$ 3276, 1999.

Elkington, S. R., Hudson, M. K., and Chan, A. A.: Resonant acceleration and diffusion of outer zone electrons in a asymmetric geomagnetic field, J. Geophys. Res., 108, doi:10.1029/2001JA009202, 2003.

Kabin, K., Rankin, R., Waters, C. L., Marchand, R., Donovan, E. F., and Samson, J. C.: Different eigenproblem models for field line resonances in cold plasma: effect on magnetospheric density estimates, Planet. Space Sci., in press, doi:10.1016/j.pss.2006.03.014, 2007.

Mann, I. R. and Wright, A. N.: Finite lifetimes of ideal poloidal Alfvén waves, J. Geophys. Res., 100, 23 677-23 686, 1995.

Mann, I. R., Wright, A. N., and Cally, P. S.: Coupling of magnetospheric cavity modes to field line resonances: A study of resonance widths, J. Geophys. Res., 100, 19441-19456, 1995.

Mathie, R. A. and Mann, I. R.: A correlation between extended intervals of ULF wave power and storm-time geosynchronous 
relativistic electron flux enhancements, Geophys. Res. Lett., 27, 3261-3264, 2000.

Northrop, T. G.: The adiabatic motion of charged particles, Interscience publishers, New York, 1963.

O'Brien, T., Lorentzen, K. R., Mann, I. R., Meredith, N. P., Blake, J. B., Fennell, J. F., Looper, M. D., Milling, D. K., and Anderson, R. R.: Energization of relativistic electrons in the presence of ULF power and MeV microbursts: Evidence for dual ULF and VLF acceleration, J. Geophys. Res., 108, doi:10.1029/2002JA009784, 2003.

Orr, D. and Matthew, J. A. D.: The variation of geomagnetic micropulsation periods with latitude and the plasmapause, Planet. Space Sci., 19, 897-905, 1971.

Radoski, H. R.: Highly asymmetric MHD Resonances: the guided poloidal mode, J. Geophys. Res., 72, 4026-4027, 1967.

Rankin, R., Fenrich, F., and Tikhonchuk, V. T.: Shear Alfvén waves on stretched magnetic field lines near midnight in Earth's magnetosphere, Geophys. Res. Lett., 27, 3265-3268, 2000.

Rankin, R., Kabin, K., Lu, J. Y., Mann, I. R., Marchand, R., Rae, I. J., Tikhonchuk, V. T., and Donovan, E. F.: Magnetospheric field-line resonances: Ground-based observations and modeling, J. Geophys. Res., 110, doi:10.1029/2004JA010919, 2005.
Rankin, R., Kabin, K., and Marchand, R.: Alfvénic Field Line resonances in arbitrary magnetic field topology, Adv. Space Res., 38, 1720-1729, 2006.

Rostoker, G., Skone, S., and Baker, D. N.: On the origin of relativistic electrons in the magnetosphere associated with some geomagnetic storms, Geophys. Res. Lett., 25, 3701-3704, 1998.

Salat, A. and Tataronis, J. A.: Conditions for existence of orthogonal coordinate systems oriented by magnetic field lines, J. Geophys. Res., 105, 13 055-13 062, 2000.

Schulz, M. and Lanzerotti, L. J.: Particle diffusion in the radiation belts, Springer-Verlag, New York, 1974.

Singer, H. J., Southwood, D. J., Walker, R. J., and Kivelson, M. G.: Alfvén-wave resonances in a realistic magnetospheric magnetic field geometry, J. Geophys. Res., 86, 4589-4596, 1981.

Stern, D. P.: Euler potentials, Amer. J. Phys., 38, 494-501, 1970.

Walker, A. D. M.: Modelling of Pc5 pulsation structure in the magnetosphere, Planet. Space Sci., 28, 213-223, 1980.

Waters, C. L., Samson, J. C., and Donovan, E. F.: Variation of plasmatrough density derived from magnetospheric field line resonances, J. Geophys. Res., 101, 24 737-24 745, 1996. 